\title{
Modèles aléatoires pour l'inférence de l'évolution du vivant / Stochastic models for the inference of life evolution (SMILE)
}

Centre interdisciplinaire de recherche en biologie (CIRB)

\section{Amaury Lambert}

\section{OpenEdition}

\section{Journals}

Édition électronique

URL : https://journals.openedition.org/annuaire-cdf/16139

DOI : 10.4000/annuaire-cdf.16139

ISBN : 978-2-7226-0572-5

ISSN : 2109-9227

Éditeur

Collège de France

\section{Édition imprimée}

Date de publication : 30 décembre 2020

Pagination : 653-657

ISBN : 978-2-7226-0516-9

ISSN : 0069-5580

\section{Référence électronique}

Amaury Lambert, « Modèles aléatoires pour l'inférence de l'évolution du vivant / Stochastic models for the inference of life evolution (SMILE) », L'annuaire du Collège de France [En ligne], $118 \mid 2020$, mis en ligne le 01 avril 2021, consulté le 22 août 2022. URL : http://journals.openedition.org/annuaire-cdf/16139 ; DOI : https://doi.org/10.4000/annuaire-cdf.16139 
Latroche C., Weiss-Gayet M., Muller L., Gitiaux C., Leblanc P., Liot S., BenLarbi S., Abou-Khalil R., Verger N., Bardot P., Magnan M., Chrétien F., Mounier R., Germain S. et ChAZAud B., "Coupling between myogenesis and angiogenesis during skeletal muscle regeneration is stimulated by restorative macrophages », Stem Cell Reports, vol. 9, no 6, 2017, p. 2018-2033, DOI : 10.1016/j.stemcr.2017.10.027.

Gitiaux C., Latroche C., Weiss-Gayet M., Rodero M.P., Duffy D., Bader Meunier B., Glorion C., Nusbaum P., Bodemer C., Mouchiroud G., Chelly J., Germain S., DEsGuerre I. et ChaZAud B., «Myogenic progenitor cells exhibit IFN type I-driven proangiogenic properties and molecular signature during juvenile dermatomyositis », Arthritis \& Rheumatology, vol. 70, $\mathrm{n}^{\circ}$ 1, 2018, p. 134-145, DOI : 10.1002/art.40328.

\section{ÉVOLUTION ET DÉVELOPPEMENT DES CELLULES GERMINALES / EVOLUTION AND DEVELOPMENT OF GERM CELLS}

Responsable : Jean-René HuYNH

\section{RECHERCHE}

Pages web : https://www.college-de-france.fr/site/en-cirb/huynh.htm et http://germcells.fr.

\section{Publication}

Clémot M., Molla-Herman A., Mathieu J., Huynh J.-R. et Dostatni N., «The replicative histone chaperone CAF1 is essential for the maintenance of identity and genome integrity in adult stem cells », Development, vol. 145, n 17,2018 , DOI : 10.1242/dev.161190.

\section{MODĖLES ALÉATOIRES POUR L'INFÉRENCE DE L'ÉVOLUTION DU VIVANT / STOCHASTIC MODELS FOR THE INFERENCE OF LIFE EVOLUTION (SMILE)}

Responsable : Amaury LAMBERT

\section{RECHERCHE}

Page web : https://www.college-de-france.fr/site/en-cirb/lambert.htm.

Dirigée par Amaury Lambert (professeur à Sorbonne Université), l'équipe SMILE regroupe des mathématiciens et des biologistes de l'évolution (quatre chercheurs titulaires et huit non titulaires). 
L'objectif général du groupe SMILE est de comprendre, de modéliser et d'inférer les processus évolutifs en distinguant trois échelles de temps :

1) l'échelle de temps micro-évolutive (démographie, extinctions);

2) l'échelle de temps méso-évolutive (différenciation des populations pouvant aller jusqu'à la spéciation) ;

3) l'échelle de temps macro-évolutive (diversification des espèces).

Nous nous intéressons en particulier à l'évolution des pathogènes, notamment depuis l'arrivée de François Blanquart (CNRS) dans l'équipe.

\section{1) Échelle micro-évolutive}

Nous étudions depuis quelques années la génétique des populations branchantes, dans le but de détecter la présence de sélection naturelle ou de démographie dans les données génomiques. Cette année, nous avons obtenu de nouveaux résultats concernant la structure d'un échantillon uniforme de taille fixée dans une population en expansion (Lambert, Theoret. Pop. Biol., 2018), une décomposition du spectre de fréquence des mutations en fonction de la position des mutations dans l'arbre généalogique qui permet de comprendre l'influence de cet arbre sur les données (Ferretti et al., Genetics, 2017), et une nouvelle méthode d'inférence sous un modèle combinant démographie hors d'équilibre et sélection (Matuszewki et al., Genetics, 2018).

Prolongements mathématiques. Nous avons étudié la structure allélique d'une grande population par un arbre continu muni d'une mesure de masse, en présence de mutations poissoniennes (Duchamps et Lambert, Ann. Appl. Probab., 2018) et nous avons partiellement identifié le processus des pertes successives, par dérive génétique, des types présents initialement dans une population (Achaz et al., J. Appl. Probab., à paraître).

\section{2) Échelle méso-évolutive}

Afin de comprendre la façon dont se forment les espèces à partir de l'accumulation de mutations responsables de l'isolement reproducteur, nous avons étudié l'état d'équilibre stochastique d'un processus à valeurs dans les graphes aléatoires, dont les arêtes modélisent l'interfertilité entre populations (Bienvenu et al., Stoch. Process. Their Appl. 2018, à paraitre).

G. Achaz a contribué à des recherches plus appliquées sur cette échelle de temps, chez la levure : la compréhension des processus d'apparition de novo (à partir de séquence non codante) de gènes, qui sont généralement petits, riches en GC et faiblement transcrits (Vakirlis et al., Mol. Biol. Evol., 2018) et l'identification de nouveaux processus mutationnels en l'absence de division cellulaire, où les insertions-délétions sont plus fréquentes qu'au cours des divisions (Gangloff et al., eLife 2017).

Prolongements mathématiques. Grâce au contour des arbres de Crump-ModeJagers, E. Schertzer et F. Simatos ont postulé de ranger leurs limites d'échelle en trois classes d'universalité, dont la première est constituée des arbres de Lévy (Schertzer et Simatos, Electronic Journal of Probability, 2018). En démontrant l'invariance des excursions des processus de Lévy réfléchis par certaines transformations trajectorielles dites « de rotation », M. Dávila Felipe et A. Lambert 
ont caractérisé le comportement de ces généalogies continues au voisinage de leur extinction (Dávila Felipe et Lambert, Electronic Journal of Probability, 2018).

\section{3) Échelle macro-évolutive}

Nous avons construit un cadre général permettant d'analyser et d'inférer les situations où des caractères quantitatifs évoluent de concert dans des espèces différentes, suivant un processus gaussien (Manceau et al., Systematic Biology, 2017).

Nous avons utilisé un modèle individu-centré de radiation adaptative pour analyser in silico la façon dont les taux de diversification (spéciation et extinction) dépendent de la diversité présente. Nos résultats montrent que la diversification d'un clade se fait en trois étapes: 1) taux constants, 2) ralentissement graduel du taux de spéciation, 3) explosion du taux d'extinction au-delà d'une capacité maximale (Aguilée et al., Nature Commun., 2018).

Dans le but de comprendre s'il existe un signal phylogénétique du risque d'extinction, nous avons proposé un nouveau modèle à trois paramètres réglant le déséquilibre de l'arbre phylogénétique ainsi que l'âge et le risque d'extinction de ses plus petits sous-arbres par rapport à ses plus grands sous-arbres. Nos résultats indiquent que les plus petits clades sont statistiquement les plus âgés et concentrent les plus grands risques d'extinction par espèce, nous amenant à craindre une perte rapide de diversité phylogénétique dans les décennies à venir (Maliet et al., Systematic Biology, à paraître).

Prolongements mathématiques. Les cours donnés par A. Lambert sur les modèles probabilistes pour les arbres phylogénétiques ont donné lieu à publication de deux articles de survol (Lambert, Brazilian Journal of Probability, 2017 ; Lambert, ESAIM Proceedings \& Surveys, 2018). Inspirés par la phylogénétique, où les généalogies de gènes sont emboîtées dans les phylogénies d'espèces, nous avons identifié tous les coalescents emboîtés markoviens (Blancas et al., Electronic Journal of Probability, 2018).

\section{4) Épidémiologie évolutive}

Nos résultats dans cette partie proviennent principalement des travaux de François Blanquart et de ses collaborateurs. Ceux-ci ont notamment montré que la coexistence entre souches sensibles et résistantes aux antibiotiques peut être expliquée par des interactions avec des gènes contrôlant la durée de portage de la bactérie. Chez Streptococcus pneumoniae, l'association prédite entre la résistance et les sérotypes conférant une durée de portage plus longue est vérifiée (Lehtinen et al., PNAS, 2017).

En utilisant un ensemble de données de 2028 patients infectés par le VIH entre 1985 et 2013 provenant de cinq pays européens, ils ont estimé l'héritabilité de la charge virale de patient à patient à environ $30 \%$ (Blanquart et al., PLoS Biology 2017).

Ils ont également développé un modèle décrivant l'évolution de la résistance aux antibiotiques et ont révélé ainsi l'existence d'une sélection stabilisatrice exercée sur la résistance aux antibiotiques, typiquement 2 à 5 fois plus forte que la sélection directionnelle pour cette résistance (Blanquart et al., Proc. Biol. Sci., 2017).

Prolongements mathématiques. Dans deux articles parus dans la revue Interface, nous avons étudié par des modèles mathématiques les conditions favorisant l'émergence d'une souche résistante aux antibiotiques (Blanquart et al., J. Roy. Soc. 
Interface, 2018) et l'issue de la compétition entre deux souches dans de petites populations hôtes (Parsons et al., J. Roy. Soc. Interface, à paraître).

\section{PUBLICATIONS}

Blancas A., Duchamps J.-J., LAMBert A. et SiRI-JÉGousse A., « Trees within trees: Simple nested coalescents », Electronic Journal of Probability, vol. 23, 2018, 94, DOI : 10.1214/18EJP219 [arXiv: 1803.02133].

FELIPE M.D. et LAMBERT A., «Branching processes seen from their extinction time via path decompositions of reflected Lévy processes », Electronic Journal of Probability, vol. 23, 2018, 98, DOI : 10.1214/18-EJP221.

Vakirlis N., Hebert A.S., Opulente D.A., Achaz G., Hittinger C.T., Fischer G., COON J.J. et LAFONTAINE I., «A molecular portrait of de novo genes in yeasts », Molecular Biology and Evolution, vol. 35, no 3, 2018, p. 631-645, DOI : 10.1093/molbev/msx315.

Landoulsi Z., LaAtar F., Noé E., Mrabet S., Ben Djebara M., Achaz G., Nava C., BAUlaC S., KACEM I., GARGOURI-BERRECHID A., GOUIDER R. et LEGUERN E., « Clinical and genetic study of Tunisian families with genetic generalized epilepsy: Contribution of CACNA1H and MAST4 genes », Neurogenetics, vol. 19, $\mathrm{n}^{\circ} 3$, 2018, p. 165-178, DOI : 10.1007/s10048-018-0550-z.

ACHAZ G., « 4 - Which model(s) explain biodiversity? », in P. GRANDCOLAS et M.-C. MAUREL (dir.), Biodiversity and Evolution, Amsterdam, Elsevier, 2018, p. 39-61, DOI : 10.1016/B978-1-78548-277-9.50004-8.

SCHERTZER E. et SiMATOS F., « Height and contour processes of Crump-Mode-Jagers forests (I): General distribution and scaling limits in the case of short edges », Electronic Journal of Probability, vol. 23, 2018, p. 67, DOI : 10.1214/18-EJP151.

*Blanquart F., Lehtinen S., Lipsitch M. et Fraser C., « The evolution of antibiotic resistance in a structured host population », Journal of the Royal Society, Interface, vol. 15, $n^{\text {o }} 143,2018$, DOI : 10.1098/rsif.2018.0040.

LAMBERT A., «The coalescent of a sample from a binary branching process », Theoretical Population Biology, vol. 122, 2018, p. 30-35, DOI : 10.1016/j.tpb.2018.04.005.

DUCHAMPS J.-J. et LAMBERT A., «Mutations on a random binary tree with measured boundary », The Annals of Applied Probability, vol. 28, no 4, 2018, p. 2141-2187, DOI : 10.1214/17-AAP1353.

Aguilée R., GASCUEl F., LAMBERT A. et FERrIERE R., « Clade diversification dynamics and the biotic and abiotic controls of speciation and extinction rates ", Nature Communications, vol. 9, no 1, 2018, p. 3013, DOI : 10.1038/s41467-018-05419-7.

LAMBERT A., «Random ultrametric trees and applications », ESAIM: Proceedings and Surveys, vol. 60, 2017, p. 70-89, DOI : 10.1051/proc/201760070.

SCHERTZER E., «Renewal structure of the Brownian taut string », Stochastic Processes and their Applications, vol. 128, no 2, 2018, p. 487-504, DOI : 10.1016/j.spa.2017.05.004.

Bridel S., Olsen A.-B., Nilsen H., Bernardet J.-F., Achaz G., Avendaño-Herrera R. et Duchaud E., "Comparative genomics of tenacibaculum dicentrarchi and "Tenacibaculum finnmarkense" highlights intricate evolution of fish-pathogenic species », Genome Biology and Evolution, vol. 10, no 2, 2018, p. 452-457, DOI : 10.1093/gbe/evy020.

Matuszewski S., Hildebrandt M.E., AChaz G. et Jensen J.D., « Coalescent processes with skewed offspring distributions and nonequilibrium demography », Genetics, vol. 208, $\mathrm{n}^{\mathrm{o}}$ 1, 2018, p. 323-338, DOI : 10.1534/genetics.117.300498.

Bienvenu F., AKÇAy E., Legendre S. et MCCAndlish D.M., «The genealogical decomposition of a matrix population model with applications to the aggregation of stages », 
Theoretical Population Biology, vol. 115, 2017, p. 69-80, DOI : 10.1016/j.tpb.2017.04.002. Ferretti L., LedDA A., WieHE T., ACHAZ G. et Ramos-Onsins S.E., « Decomposing the site frequency spectrum: The impact of tree topology on neutrality tests », Genetics, vol. 207, n 1 , 2017 , p. 229-240, DOI : 10.1534/genetics.116.188763.

Gangloff S., Achaz G., Francesconi S., Villain A., Miled S., Denis C. et ARCANGIOLI B., « Quiescence unveils a novel mutational force in fission yeast », ELife, vol. 6, 2017, DOI : 10.7554/eLife.27469.

Blanquart F., Wymant C., Cornelissen M., Gall A., Bakker M., Bezemer D., Hall M., Hillebregt M., Ong S.H., Albert J., Bannert N., Fellay J., Fransen K., Gourlay A.J., GRABOWSKI M.K., GUNSENHEIMER-BARTMEYER B., GÜNTHARD H.F., KiVelä P., Kouyos R., LAEYendecKer O., LitTSOla K., MEYER L., PORTER K., Ristola M., VAn Sighem A., Vanham G., Berkhout B., Kellam P., Reiss P., Fraser C. et BEEHIVE COLLABORATION, "Viral genetic variation accounts for a third of variability in HIV-1 setpoint viral load in Europe », PLoS biology, vol. 15, n 6, 2017, e2001855, DOI : 10.1371/ journal.pbio.2001855.

*Blanquart F., Lehtinen S. et Fraser C., « An evolutionary model to predict the frequency of antibiotic resistance under seasonal antibiotic use, and an application to Streptococcus pneumoniae », Proceedings. Biological Sciences, vol. 284, $\mathrm{n}^{\circ}$ 1855, 2017, DOI : 10.1098/ rspb.2017.0679.

*Lehtinen S., Blanquart F., Croucher N.J., Turner P., Lipsitch M. et Fraser C., «Evolution of antibiotic resistance is linked to any genetic mechanism affecting bacterial duration of carriage », Proceedings of the National Academy of Sciences of the United States of America, vol. 114, no 5, 2017, p. 1075-1080, DOI : 10.1073/pnas.1617849114.

BAILEY S.F., BLANQUART F., BATAILLON T. et KASSEN R., « What drives parallel evolution?: How population size and mutational variation contribute to repeated evolution », BioEssays: News and Reviews in Molecular, Cellular and Developmental Biology, vol. 39, $\mathrm{n}^{\circ}$ 1, 2017, p. 1-9, DOI : 10.1002/bies.201600176.

PARSOnS T.L., LAMBERT A., DAY T. et GANDON S., « Pathogen evolution in finite populations: slow and steady spreads the best », Journal of the Royal Society, Interface, vol. 15, $\mathrm{n}^{\circ} 147$, 2018, DOI : 10.1098/rsif.2018.0135.

SCHERTZER E. et STAVER A.C., « Fire spread and the issue of community-level selection in the evolution of flammability », Journal of the Royal Society, Interface, vol. 15, $\mathrm{n}^{\circ}$ 147, 2018, DOI : 10.1098/rsif.2018.0444.

Maliet O., Gascuel F. et LAMBERT A., «Ranked tree shapes, nonrandom extinctions, and the loss of phylogenetic diversity ", Systematic Biology, vol. 67, n 6, 2018, p. 1025-1040, DOI : 10.1093/sysbio/syy030.

LAMBERT A. et SCHERTZER E., « Recovering the Brownian coalescent point process from the Kingman coalescent by conditional sampling », Bernoulli, [sous presse], http://arxiv.org/ abs/1611.01323.

LAmbert A., PinA V.M. et SChERTZER E., Chromosome Painting, 2018, https://hal.archivesouvertes.fr/hal-01850398. 